\title{
Message from the Editors of Hernia
}

\author{
G. Campanelli ${ }^{1}$
}

Published online: 19 February 2019

c) Springer-Verlag France SAS, part of Springer Nature 2019

Dear Hernia Friends, Dear Hernia Readers,
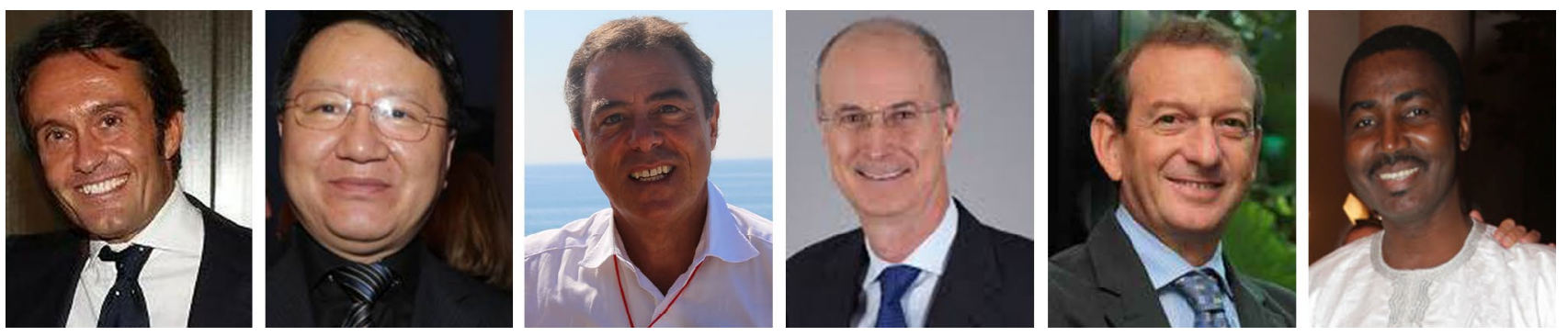

It's a great pleasure for me to announce that starting January 2019, Hernia will be supported by a renewed Editorial Board, comprising five Associate Editors, $40 \mathrm{Sec}-$ tion Editors and a broad International Advisory Board.

Many members serving on the previous editorial board accepted to renew their commitment to the journal; they are very welcome and I am sure that their experience will represent an added value to Hernia. The complete Editorial Board is already online at https://www.springer.com/medic ine/surgery/journal/10029/PSE?detailsPage=editorialBoard.

With the cooperation of these outstanding colleagues and of new excellent members, including many enthusiastic young surgeons, the Journal will continue to offer rigorous peer-review and nothing will change in the way manuscripts are handled.

Moreover, a very active and efficient Editorial Office will support you and Authors submitting to the Journal during the peer-review process.

I am very proud and excited to serve as Editor-in-Chief and to take over this responsibility from my predecessors Marc Miserez, Robert Fitzgibbons, their Associates and

G. Campanelli

giampiero.campanelli@grupposandonato.it

1 University of Insubria, Gruppo Ospedaliero San Donato, Milan, Italy those who represented the milestones of hernia surgery: Jean Paul Chevrel, Charles J. Filipi, Andrew Kingsnorth, Robert Bendavid, Volker Schumpelick.

The American Hernia Society, the European Hernia Society and the Asia-Pacific Hernia Society three societies with whom I had a long-lasting cooperation as EHS Secretary General for more than 14 years and then EHS President, continue to be affiliated to the Journal. The first world conference, that was held 3 years ago and endorsed all the hernia societies, was the result of this close cooperation and I am sure that the Journal will provide a further opportunity to strengthen the current connections among these societies.

The vision for the Journal that I share with the Associate Editors is to create a "working platform", a forum where worldwide surgeons can start a discussion, send suggestions, propose new techniques and new ideas on any cutting-edge topics in the hernia daily activities.

To this end, we will invite well-known experts to submit systematic reviews on the more debated aspects of hernia surgery, but we will also encourage all the Hernia friends to submit their research to the Journal for our thorough evaluation.

The Journal welcomes papers on daily hernia surgical activities as well as papers dealing with more complex cases such as reconstructions of the abdominal wall. Moreover, Hernia encourages submissions focusing on 
multi recurrences, chronic pain, pubic inguinal pain syndrome, diastasis recti and floppy abdomen, emergency issues and developmental studies on new (and "old") exciting approaches, such as robotic, minimally invasive lap and open surgery; new approaches suggested by plastic surgeons are also of interest. New ideas, new techniques, criticisms as well as teaching reports of failure and complications will be more than welcome.

One of the main aim for us will be the speed of publication, that is of utmost importance. In fact, another objective that we plan to reach is a considerable decrease in the turnaround times from submission to first decision. We will work closely with the Editorial Office, the Section Editors and the Reviewers to make this happen.

A new "Editor's Corner" focusing on hot topics will be launched since the first 2019 issue. Associate Editors and myself will propose to the readers to start a discussion on these topics, thus creating a forum on specific matters. The themes of the discussion forum will be: primary unilateral inguinal hernia (non-complicated), primary ventral hernias, femoral hernias, incisional (open vs laparoscopic $v s$ robotic), indications and results of all kind of component separation and diastasis recti. We will invite papers and look forward to receiving additional submissions on these specific topics from you, to trigger a lively discussion.

The primary focus of the Journal is to build on its existing reputation by further improving the quality of the published articles. In this regard, Reviewers and Editors are asked to thoroughly assess the assigned manuscripts, provide detailed comments and highly valuable suggestions to the Authors.

This vision can only be accomplished if you act as a protagonist and support Hernia by sending your papers. Rigorous scientific approach, perfect technical support and empathy with the patient will be our guide in the years to come.

We look forward to your comments, suggestion, and above all, your contribution to the Journal.

\section{Giampiero Campanelli}

Editor in Chief

Jie Chen

Diego Cuccurullo

Namir Katkhouda

Davide Lomanto

Rachid Sani

Associate Editors

Silvia Malosio

Managing Editor

Publisher's Note Springer Nature remains neutral with regard to jurisdictional claims in published maps and institutional affiliations. 